Check for updates

Cite this: J. Mater. Chem. C, 2019, 7,256

Received 31st August 2018, Accepted 3rd December 2018

DOI: $10.1039 / c 8 t c 04368 j$

rsc.li/materials-c

\title{
Tailorable, 3D structured and micro-patternable ionogels for flexible and stretchable electrochemical devices $\dagger$
}

\author{
Yong Zhong, (D) ab Giao T. M. Nguyen, ${ }^{b}$ Cédric Plesse, $\mathbb{D}^{\mathrm{b}}$ Frédéric Vidal $\mathbb{D}^{\mathrm{b}}$ and \\ Edwin W. H. Jager (D)*a
}

\begin{abstract}
A new family of ionogels for electrochemical devices was developed from a mixture of multifunctional thiols, diacrylate and triethylamine in the presence of ionic liquid using Michael addition chemistry. Polymerization kinetic studies show that the ionic liquid not only acts as an ion source but also a co-catalyst in the polymerization. lonogels with tailorable surface and mechanical properties were prepared using three approaches: off-stoichiometry, methacrylate addition, and dithiol chain extender addition. 3-Dimensional ionogels were constructed by bonding the flexible ionogel film together using the ionogel solution as an ionic adhesive. A tube actuator with PEDOT-PSS patterned on inner and outer wall was prepared to illustrate the potential of these ionogels with reactive surfaces. In addition, micro-patterns of the ionogels were obtained by photolithography and soft imprinting lithography. All in all, this thiol acrylate Michael chemistry provides a platform to prepare various forms (films, micro-patterns, 3-dimensional structures, and adhesive) of ionogels for the next generation of flexible electrochemical devices.
\end{abstract}

\section{Introduction}

The increasing demand for wearable and stretchable electrochemical devices has emphasized a need for flexible and stretchable polymer gel electrolytes. Polymer gel electrolytes, i.e. percolated polymer gel networks with liquid electrolytes, ${ }^{1}$ are of great interest as an attractive alternative to liquid electrolyte systems. Polymer gel electrolytes not only overcome the leakage, toxicity and flammability issues related to liquid electrolytes, but also offer other attractive properties, such as thin-film forming ability, flexibility, stretchability, transparency and relatively high ionic conductivity. ${ }^{2}$ Polymer gel electrolytes are used in electrolyte-gated transistors, ${ }^{3}$ supercapacitors, ${ }^{4}$ dielectric elastomer transducers, ${ }^{1}$ electrochromic devices, ${ }^{5}$ ionic actuators, ${ }^{6}$ solar cells, ${ }^{7}$ electrochemical sensors, ${ }^{8}$ batteries, ${ }^{9}$ and other electrochemical devices. Of the various liquid electrolytes used in polymer gel electrolytes, ionic liquids, also known as room-temperature molten salts, have received significant attention because of their unique combination of physicochemical properties, such as very low vapor pressure, non-flammability, good chemical and thermal stability, large

\footnotetext{
${ }^{a}$ Sensor and Actuator Systems (SAS), Department of Physics, Chemistry and Biology (IFM), Linköping University, Linköping, Sweden. E-mail: edwin.jager@liu.se

${ }^{b}$ Laboratoire de Physicochimie des Polymères et des Interfaces, Institut des Matériaux, Université de Cergy-Pontoise, Cergy-Pontoise Cedex, France

$\dagger$ Electronic supplementary information (ESI) available. See DOI: 10.1039/c8tc04368j
}

electrochemical window, and high ionic conductivity. ${ }^{2,10}$ Such polymer gel electrolytes based on ionic liquids, so called ionogels, were either achieved by in situ gel network formation in an ionic liquid or by swelling a polymer gel network with ionic liquids. Various gel techniques have been used to synthesize the supporting polymer gel network, which leads to a variety of ionogels with different properties. Among them, in situ free radical photo-polymerization of acrylate monomers in ionic liquids yields highly conductive polymer electrolyte films in a short polymerization time due to its high energy efficiency. ${ }^{2}$ It has also been reported that photo-polymerization allows the preparation of micro-patterns of gel electrolytes, which are essential in the fabrication of microdevices. ${ }^{11,12}$ Incorporating ionic liquid into a structurally homogeneous polymer network prepared from tetra-arm poly(ethylene glycol) leads to a highly ion-conducting polymer electrolytes with ionic conductivities nearly equal to those of pure ionic liquids. ${ }^{13}$ Polymer networks with a semi- or full interpenetrating polymer network structure have also been used, which leads to gel polymer electrolytes with enhanced mechanical properties. ${ }^{14}$

A need exists for tuneable polymer gel electrolyte systems in which not only the mechanical properties but also the surface properties are tailorable. Tailorable mechanical properties are required to meet the exact requirements for applications in flexible and stretchable devices fabrication. Ionogels with reactive surfaces are extremely useful to enhance the adhesion between 
the different layers in multi-layered devices, which is a common structure in most electrochemical devices. In addition, to miniaturize or fabricate microarrays of these gel electrolytes-based devices, micro-patternable polymer gel electrolytes are needed. To meet those demands for polymer gel electrolytes, novel polymerization network formation routes through which the gel electrolyte can be made, need to be devised.

The thiol Michael addition polymerization system has received significant attention in the field of polymer science in the last decades. ${ }^{15,16}$ It is a highly versatile reaction involving a base or nucleophile-catalysed addition of a thiolate anion to electrondeficient alkenes such as maleimides, vinyl sulfones, acrylates, and methacrylates. Classified as a 'click' reaction, the thiol Michael addition reaction is highly efficient, modular in nature, and exhibits a nearly ideal 1:1 stoichiometric reactivity. ${ }^{17}$ It proceeds without heat or light exposure by using minimal amounts of base or nucleophilic catalysts at room temperature. Over the past decades, this reaction has been utilized by many researchers in a range of applications, including bioconjugation, small molecule synthesis, surface functionalization, tissue engineering, polymer network synthesis, and dendrimer synthesis. ${ }^{18}$

Here, we employed the Michael addition reaction as a platform to develop ionogels with tuneable mechanical and surface properties. Kinetics of the polymer network formation, in situ in an ionic liquid, were studied and the result shows that the ionic liquid acts a co-catalyst in the gel formation. We utilised the reactive surfaces to construct 3-dimensional ionogels with a uniform structure by folding and bonding flexible ionogel film together using the as-prepared ionogel solution as an ionic conducting adhesive. To demonstrate the potential of the new ionogels, we utilised this folding and bonding approach to fabricate tubes and boxes that comprised patterned structures, such as conducting lines, on both the inside and outside of the shapes. A special tube actuator with PEDOT-PSS patterned on inner and outer tube wall was further prepared to illustrate the potential of ionogels with reactive surface. In addition, micropatterns of ionogels were also obtained as these materials are also compatible with standard photolithography and soft imprinting lithography. This represents an alternative path toward solid state electrolytes with various 3-dimensional shapes using thiol acrylate chemistry.

\section{Results and discussion}

The ionogel was prepared from a blend of multifunctional trithiol cross-linker (trimethylolpropane tris(3-mercoptopropianate)) and PEG diacrylate (PEGDA) in the presence of 1-ethyl3-methylimidazolium bis(trifluoromethylsulfonyl)imide (EMIM TFSI) ionic liquid using thiol acrylate Michael addition reaction with triethylamine (TEA) as a base catalyst. To begin with, the related polymerization mechanism is discussed. A thiol acrylate mixture can proceed via an ionic Michael addition mechanism with a base catalyst, which deprotonates a thiol group and creates a reactive thiolate anion. ${ }^{19}$ Addition of the thiolate anion to an electron-deficient unsaturated acrylate group generates a highly reactive carboanion, which deprotonates another thiol group for reactive thiolate anion regeneration. ${ }^{20}$ The thiol acrylate Michael addition cycle continues until one of the reactants reaches full conversion. The result of this thiol acrylate Michael addition reaction in a multifunctional trithiol and PEG diacrylate mixture is that all thiol groups and electron poor acrylate groups are perfectly alternatingly crosslinked to form a uniform and homogeneous polymer network which is otherwise difficult to obtain by conventional free radical polymerization of PEG diacrylate.

The polymerization kinetics in a stoichiometric 1:1 molar ratio of thiol to acrylate functional groups with $1 \mathrm{wt} \%$ TEA as the catalyst was first studied with FT-IR techniques by monitoring the disappearance of the thiol peak centred about $2570 \mathrm{~cm}^{-1}$ and acrylate peaks at $810 \mathrm{~cm}^{-1}$ for up to 22 hours. Fig. 2 shows the FTIR spectra for the TEA catalysed trithiol-PEG diacrylate Michael addition reactions in the absence of EMIMTFSI, i.e. in bulk. The reaction occurs spontaneously when it is mixed. The concurrent decrease in the intensity of both the thiol group and the acrylate group indicates both functional groups are participating in the polymerization. In order to study the conversion of the functional group, integration of peak area at different reaction times was performed. Thiol and acrylate conversions as a function of reaction time are shown in Fig. 3. The reactions reached 90\% conversion of both thiol and acrylate groups after about 22 hours and an equal conversion rate for thiol acrylate groups was observed, which confirms the thiol acrylate Michael proceeds via a 1-to-1 fashion between thiol and acrylate groups. It should be noted that accurate determination of thiol conversions is difficult because thiol groups present a weak absorption signal and the thiol monomers contribute less of the mass in the mixture, resulting in a small, relatively noisy thiol absorption peak especially when ionic liquid was introduced into the mixture. Therefore, acrylate conversion is used to represent the overall polymerization reaction rate when ionic liquid is added into the thiol acrylate mixture. A significant increase in the reaction rate by adding $50 \mathrm{wt} \%$ ionic liquid into the thiol acrylate mixture was observed, as also shown in Fig. 3. This leads to a higher conversion of acrylate groups $(>98 \%)$ in short time periods of around $40 \mathrm{~min}$. The acceleration of polymerization rate by adding EMIM TFSI ionic liquid can be explained as follows. The thiol Michael addition can be catalysed by a nucleophile as mentioned previously. A new class of nitrogen-centred nucleophilic catalysts including 1-methyl imidazole and imidazole for the thiol-Michael addition reactions was recently studied by Bowman and co-workers. ${ }^{21}$ They have demonstrated that imidazole and 1-methyl imidazole were extremely effective catalysts for thiol-vinylsulfone Michael addition reaction and a relatively weak catalytic effect was also observed for thiol acrylate Michael addition reaction. Direct catalytic effect of imidazolium based ionic liquid on Michael reaction without any additional catalyst was also demonstrated in several studies in the literature. ${ }^{22-24}$ For example, Michael addition of various thiophenols to chalcones proceeded smoothly in several imidazolium based ionic liquids with the yield the same or only slightly lower than when L-proline catalyst was used, which proved its nucleophilicity. ${ }^{25}$

Inspired by these reactions, we hypothesize that the EMIM TFSI ionic liquid will also catalyse the thiol-acrylate Michael 
<smiles>CCC(COC(=O)CCS)(COC(=O)CCS)COC(=O)CCS</smiles><smiles>C=CC(=O)OCC(C)(CC)OC(=O)C=C</smiles><smiles>C=C(C)C(=O)OC(C)(C)COC(C)(C)C</smiles>

(d) $\mathrm{CH}_{3}$<smiles>CCn1ccnc1</smiles><smiles>O=[Si](C(F)(F)F)S(=O)(=O)[Si](O)(F)C(F)(F)F</smiles>

(e)<smiles>O=C(CS)OCCCCOC(=O)CS</smiles>

(f)<smiles>CCN(CC)CC</smiles>

(g)

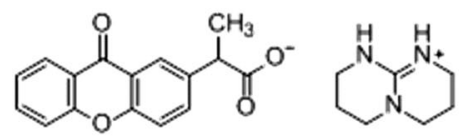

Fig. 1 Chemical structures of materials used in this study: (a) trimethylolpropane tris(3-mercoptopropianate) (TT), (b) poly(ethylene glycol)diacrylate $\left(\right.$ PEGDA, average $M_{n}=700 \mathrm{~g} \mathrm{~mol}^{-1}$ ), (c) poly(ethylene glycol)methyl ether methacrylate (PEGMA, average $M_{\mathrm{n}}=500 \mathrm{~g} \mathrm{~mol}{ }^{-1}$ ), (d) 1 -ethyl-3methylimidazolium bis(trifluoromethylsulfonyl)imide (EMIM TFSI), (e) 1,4-butanediol bis(thioglycolate)(dithiol), (f) triethylamine (TEA) and (g) 2-(9oxoxanthen-2-yl)propionic acid 1,5,7-triazabicyclo[4.4.0]dec-5-ene salt (photobase generator, PBG).

addition to some extent in a similar way. To evaluate this hypothesis, the acrylate group conversion for thiol acrylate mixture in the presence of $50 \mathrm{wt} \%$ ionic liquid without TEA addition was monitored and the acrylate group conversion versus time was plotted. In the beginning, there was no acrylate conversion but after about 5 days, around $10 \%$ of acrylate group conversion was finally observed (Fig. 3). This leads to the conclusion of the weak catalytic effect of EMIM TFSI ionic liquid on thiol acrylate Michael reaction. Thus, the addition of $50 \mathrm{wt} \%$ ionic liquid together with $1 \mathrm{wt} \%$ TEA, both weak catalysts, leads to a synergistic catalytic effect on the thiol acrylate Michael reaction, resulting in a fast polymerization kinetic (Fig. 3). This is interesting because adding solvent into a polymerizing system generally leads to a slow polymerization rate due to a dilution effect. But addition of a catalytic solvent as in this case results in a polymerizing system with faster kinetics and higher conversion of functional groups. In this polymerizing system, the ionic liquid not only acts a catalyst of the polymer network formation, but also as an ion source for the ionogel material which is formed in situ after the formation of polymer network from multifunctional trithiol crosslinker and PEG diacrylate. It was also interesting to find that the polymerization kinetics was also affected by stoichiometry. An off-stoichiometric formulation with excess thiol functionality of $37.5 \%$ had a faster polymerization rate and higher conversion in a shorter time than stoichiometric formation. This was simply due to the concentration effect on reaction rate as increasing the concentrations of the reactants will usually result in the corresponding increase in the reaction rate. Adding excess thiol group means increasing the thiol concentration in the mixture, which leads to the observed fast kinetics in an off-stoichiometric formation.

The thiol acrylate Michael addition between multifunctional trithiol and PEGDA with TEA catalyst in the presence of an ionic liquid is an efficient and fast 1:1 stoichiometric reaction.
These polymerization properties and the use of a multifunctional thiol allow us to easily control the cross-link density of the thiolacrylate polymer network in a systematic manner by varying the monomer stoichiometry, addition of methacrylate, or addition of dithiol chain extender as illustrated in Fig. 4. The resulting tuneable polymer network with the ionic liquid inside leads to ionogel with tailorable surface and mechanical properties as discussed below.

In general, stoichiometric formulations with an exact equal number of thiol and acrylate groups, achieve full conversion of reactive groups into a highly cross-linked polymer network. This is shown in Fig. 5 that there were very few or no reactive groups remaining after full curing for formulation with thiol acrylate ratio 100:100 (sample no. 1, Table 1), as evidenced by the disappearance of thiol and acrylate FT-IR peaks. However, intentional deviation from stoichiometric thiol acrylate formulations leads to a number of advantages. It enables the creation of a polymer network with a well-defined amount of residual thiol or acrylate groups after complete polymerization. The FT-IR study in Fig. 5b confirmed the presence of remaining acrylate groups after complete polymerization and the absence of thiol groups in the formulation with excess acrylate functionality of $25 \%$. In the formulation with excess thiol functionality of $25 \%$, it was not easy to resolve the remaining thiol groups from the spectra as the thiol peak was noisy and diluted by ionic liquid as shown in Fig. 5 a. However, the spectra for formulation with excess thiol functionality of $37.5 \%$ clearly showed that the resulting polymer network consists of fully reacted acrylate groups and remaining unreacted thiols group with an obvious thiol FT-IR peak. At the same time such off-stoichiometric formulations also lead to tuned mechanical properties by adjusting the off-stoichiometric ratio without changing monomer structures (Table 1). The Young's modulus deceased from 2.1 MPa (sample no. 1, Table 1) to $1.8 \mathrm{MPa}$ (sample no. 3, Table 1) as the excess thiol functionality increased from $0 \%$ to $37.5 \%$ and an even lower modulus of 

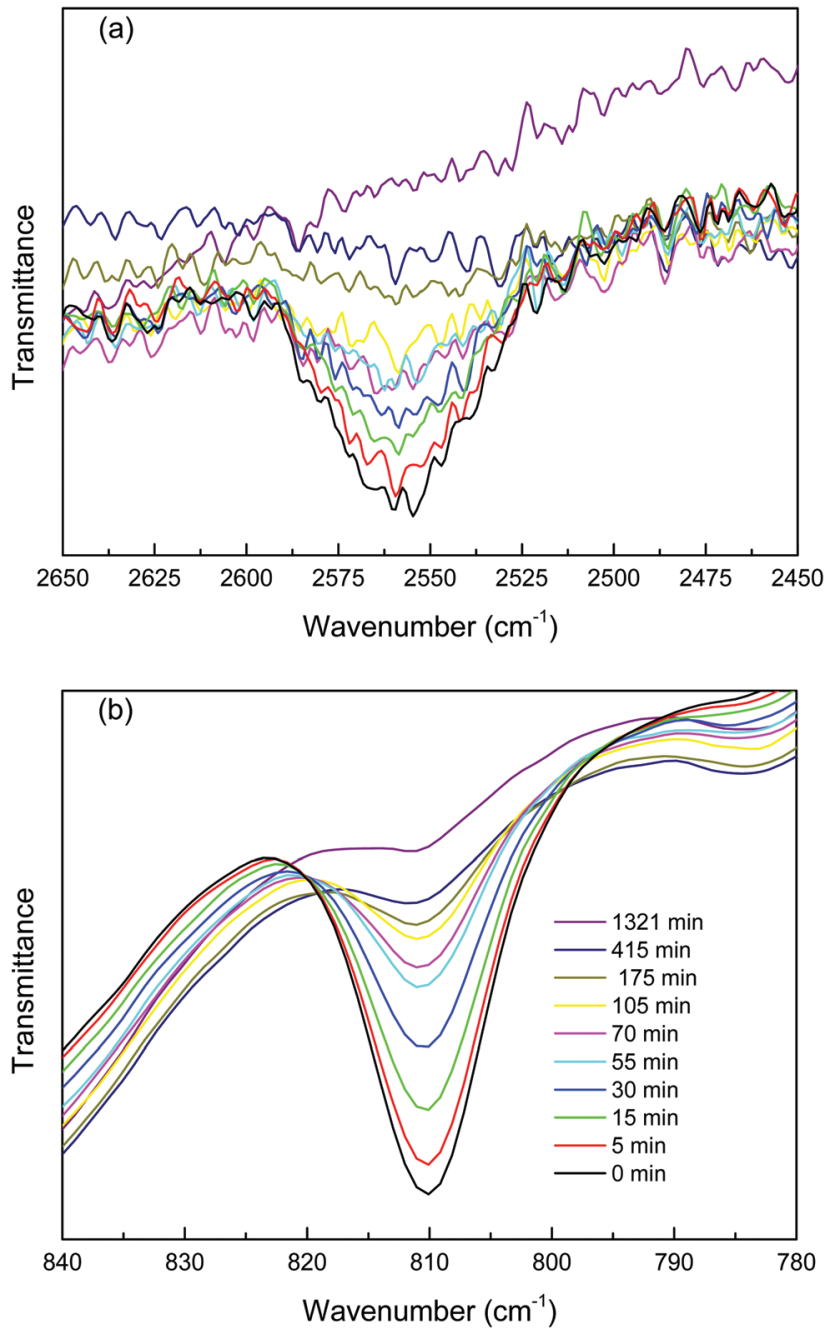

Fig. 2 FTIR spectra in transmittance for the (a) thiol and (b) acrylate peaks in a 1:1 stoichiometric mixture of thiol acrylate with $1 \mathrm{wt} \%$ of TEA showing decrease in absorbance over time indicating conversion into the gel electrolyte.

$0.43 \mathrm{MPa}$ was obtained when the excess acrylate functionality was $25 \%$ (sample no. 4, Table 1). At off-stoichiometry, fewer cross-links to the network are formed and the resulting ionogel is softer and more flexible. Herein, we clearly show that only by varying the thiol acrylate molar ratio with the same starting materials, it is possible to control the final polymer network structure and its polymerization kinetics to obtain ionogel with tailorable mechanical and surface properties. The residual thiol or acrylate groups on the surface from off-stoichiometric formulations enable the development of ionogel with reactive surface.

The mechanical and surface properties of ionogel can also be tuned by adding monofunctional PEG methacrylate into an off-stoichiometric formation by taking advantage of reactivity and concentration difference. Previous studies showed that sterically-hindered and less polarized methacrylates react nearly 200 times slower than more polarized acrylates in the thiol-Michael addition reaction. ${ }^{26}$ This kinetic differences in the rate of thiol-Michael addition reaction between acrylates

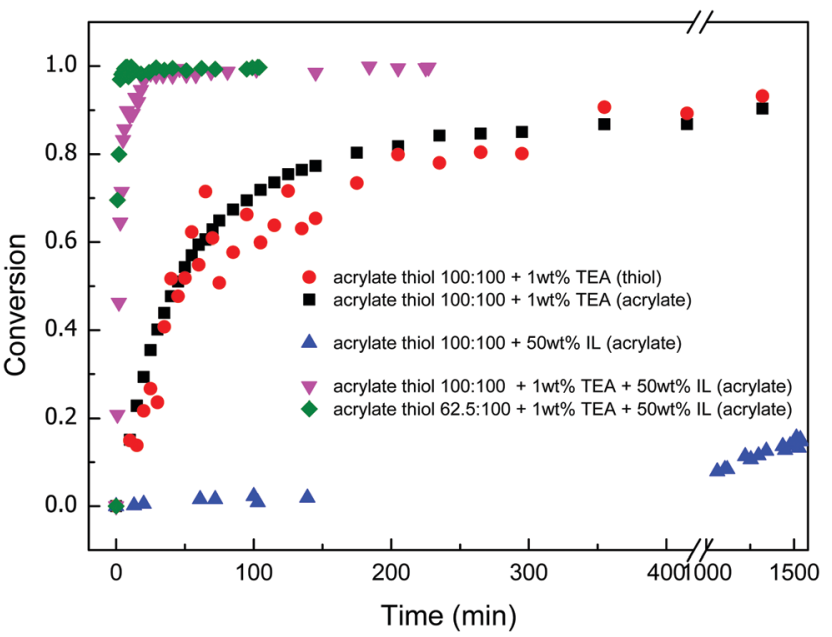

Fig. 3 Thiol and acrylate functional group conversion vs. time plots for the polymerization kinetics studies. Labels indicate the composition of each sample. Conversion is determined by monitoring the disappearance of the thiol peak at $2570 \mathrm{~cm}^{-1}$ and acrylate peak at $810 \mathrm{~cm}^{-1}$, respectively.
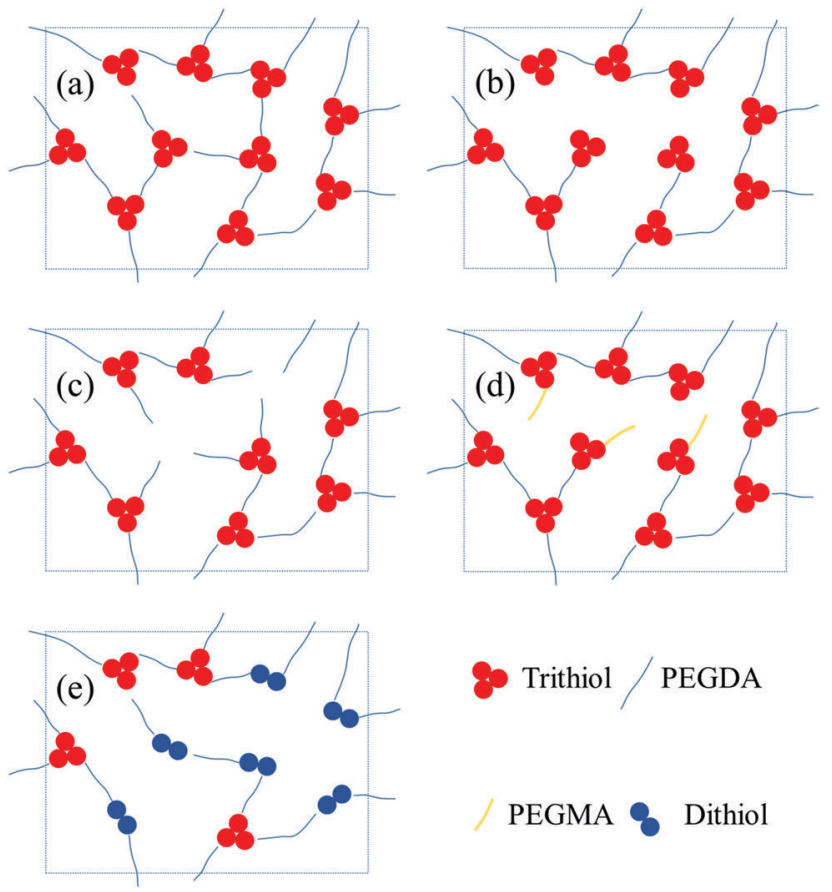

Q Trithiol / PEGDA

PEGMA 8 Dithiol

Fig. 4 Idealized network schematics for thiol acrylate formulation with (a) 1:1 thiol acrylate functional groups; (b) excess thiol groups; (c) excess acrylate groups; (d) addition of methacrylate and (e) addition of dithiol chain extender.

and methacrylates was exploited to synthesize linear segmented copolymers in a one-pot approach. ${ }^{24}$ Using the same methodology, trithiol, PEG diacrylate and PEG methacrylate in a functional groups molar ratio of $100: 75: 12.5$ in the presence of the ionic liquid and TEA was mixed (sample no. 5, Table 1). It can be expected that thiol groups react readily with acrylate groups via Michael addition in the first stage until complete consumption of PEG diacrylate to form thiol-terminated polymer network while 

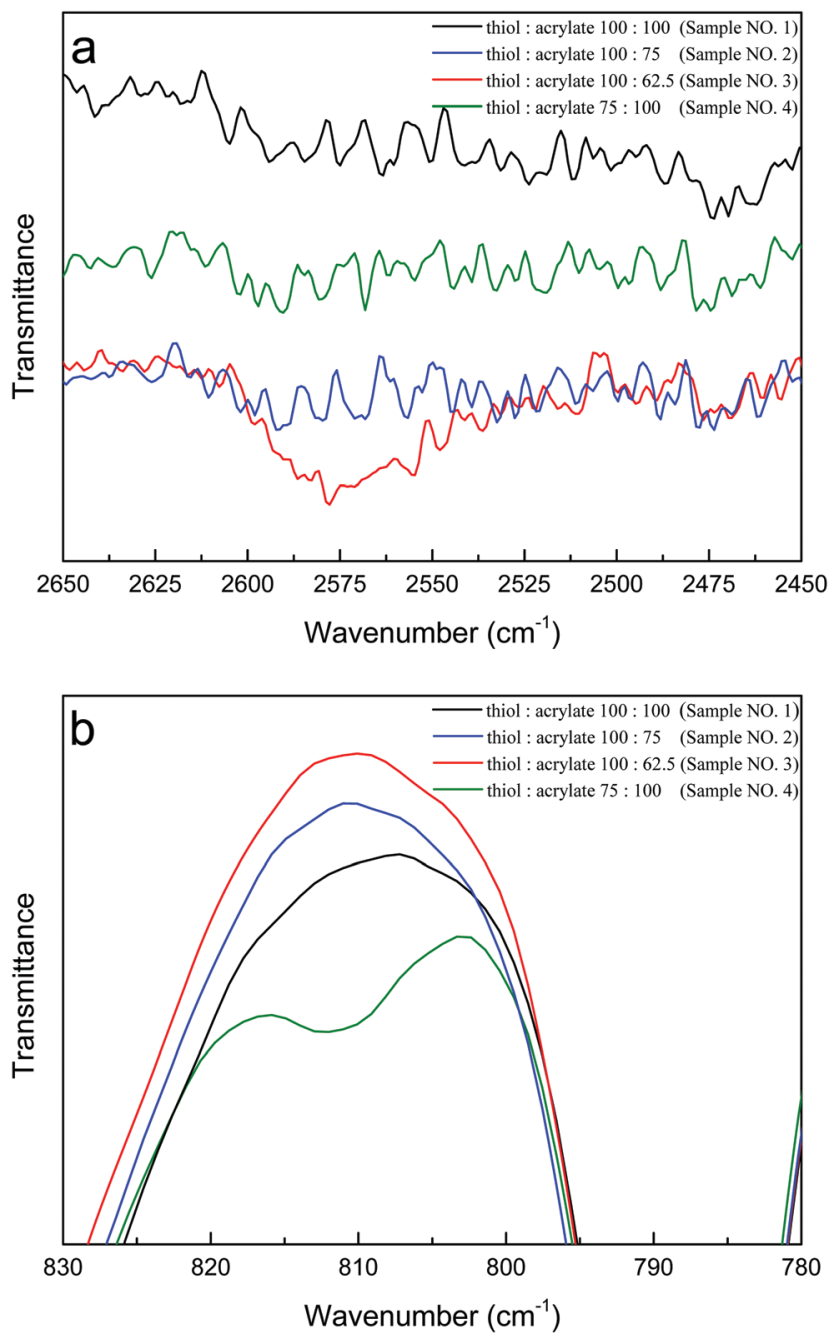

Fig. 5 FTIR spectra after completion of polymerization for (a) thiol and (b) acrylate peaks in thiol acrylate mixture (sample no. 1-4) with various stoichiometric ratios of thiol acrylate functional groups. The composition of each sample is shown in Table 1 and spectra for other formation (sample no. $5-8)$ are shown in Fig. S2 (ESI†).

leaving the methacrylate monomer virtually unreacted due to the huge reactivity and concentration difference. After extended reaction times, the methacrylate monomers react with remaining thiol groups to create dangling chains in the polymer network during the second reaction stage. The addition of $12.5 \%$ methacrylate led to a decrease in Young's modulus of the resulting ionogel from 1.9 $\mathrm{MPa}$ to $1.0 \mathrm{MPa}$ and a reduction of unreacted thiol groups. A stoichiometric mixture of trithiol, diacrylate and methacrylate with the molar ratio of $100: 75: 25$ (sample no. 6, Table 1) allowed to obtain ionogel with fully reacted thiol groups and a lower modulus of $0.9 \mathrm{MPa}$.

Another approach to control the crosslink density in the polymer network is to gradually replace the trithiols crosslinkers with dithiols in the starting material while maintaining $1: 1$ thiol acrylate stoichiometry. For the case when only trithiol monomers reacted with the diacrylate monomers, all the trifunctional monomer units serve as crosslinkers of degree 3 , leading to a highly cross-linked polymer network. The addition of dithiol monomers to this system not only leads to a decrease in the concentration of trifunctional thiol crosslinkers but also increases the molecular weight between the crosslinks as chain extenders. This approach made it possible to decrease the network crosslinking density even further, resulting in better tuning of the mechanical properties of the ionogel. Fig. 6 provides representative experimental stress strain curves for formulations with increasing percentage of dithiol. All the formulations were allowed to achieve $100 \%$ conversion of both thiol and acrylate groups. As dithiol content increased, the Young's modulus as reported in Table 1 significantly decreased, indicating a decreased cross-linking density. The Young's modulus could be decreased by nearly one order of magnitude, from 2.1 MPa (sample no. 1, Table 1) to $0.3 \mathrm{MPa}$ by replacing $75 \%$ thiol groups with dithiol extender (sample no. 8, Table 1). In addition to the effect on the Young's modulus, the elongation at break was also affected. When all the reacting thiol functional groups are from trithiol monomers, the ionogel breaks at a low strain of $17 \%$. The formulation with $50 \%$ thiol functional groups from dithiol extender (sample no. 7, Table 1) show an improvement of the fracture strain to $63 \%$. The ionogels with substantially reduced concentration of crosslinks, with 75\% thiol functional groups from dithiol extender, were considerably more stretchable, exhibiting elongation at break to $155 \%$. This strain improvement indicates that the polymer networks deviate from a densely cross-linked state to a considerably more stretchable state by using dithiol chain extender in the formulation. This property is highly desirable for applications in wearable and stretchable devices fabrication, as it enables repeated use and large deformations without failure.

Table 1 The composition of each ionogel sample and their surface, mechanical and conductivity properties

\begin{tabular}{|c|c|c|c|c|c|c|c|c|c|}
\hline \multirow[b]{2}{*}{$\begin{array}{l}\text { Sample } \\
\text { no. }\end{array}$} & \multicolumn{4}{|c|}{ Functional groups molar ratio } & \multirow[b]{2}{*}{$\begin{array}{l}\text { TEA } \\
(w t \%)\end{array}$} & \multirow[b]{2}{*}{$\begin{array}{l}\text { Ionic liquid } \\
(\mathrm{wt} \%)\end{array}$} & \multirow[b]{2}{*}{$\begin{array}{l}\text { Surface } \\
\text { properties }\end{array}$} & \multirow[b]{2}{*}{$\begin{array}{l}\text { Young's } \\
\text { modulus (MPa) }\end{array}$} & \multirow[b]{2}{*}{$\begin{array}{l}\text { Ionic conductivity } \\
\left(10^{-4} \mathrm{~S} \mathrm{~cm}^{-1}\right)\end{array}$} \\
\hline & $\begin{array}{l}\text { Thiol } \\
\text { (trithiol) }\end{array}$ & $\begin{array}{l}\text { Acrylate } \\
\text { (PEGDA) }\end{array}$ & $\begin{array}{l}\text { Methacrylate } \\
\text { (PEGMA) }\end{array}$ & $\begin{array}{l}\text { Thiol } \\
\text { (dithiol) }\end{array}$ & & & & & \\
\hline 1 & 100 & 100 & - & - & 1 & 50 & Non-reactive & 2.1 & 3.75 \\
\hline 2 & 100 & 75 & - & - & 1 & 50 & Thiol-rich & 1.9 & 5.69 \\
\hline 3 & 100 & 62.5 & - & - & 1 & 50 & Thiol-rich & 1.8 & 6.1 \\
\hline 4 & 75 & 100 & - & - & 1 & 50 & Acrylate-rich & 0.43 & 4.98 \\
\hline 5 & 100 & 75 & 12.5 & - & 1 & 50 & Thiol-rich & 1.0 & 7.12 \\
\hline 6 & 100 & 75 & 25 & - & 1 & 50 & Non-reactive & 0.9 & 7.43 \\
\hline 7 & 50 & 100 & - & 50 & 1 & 50 & Non-reactive & 0.96 & 4.89 \\
\hline 8 & 25 & 100 & - & 75 & 1 & 50 & Non-reactive & 0.30 & 6.31 \\
\hline
\end{tabular}




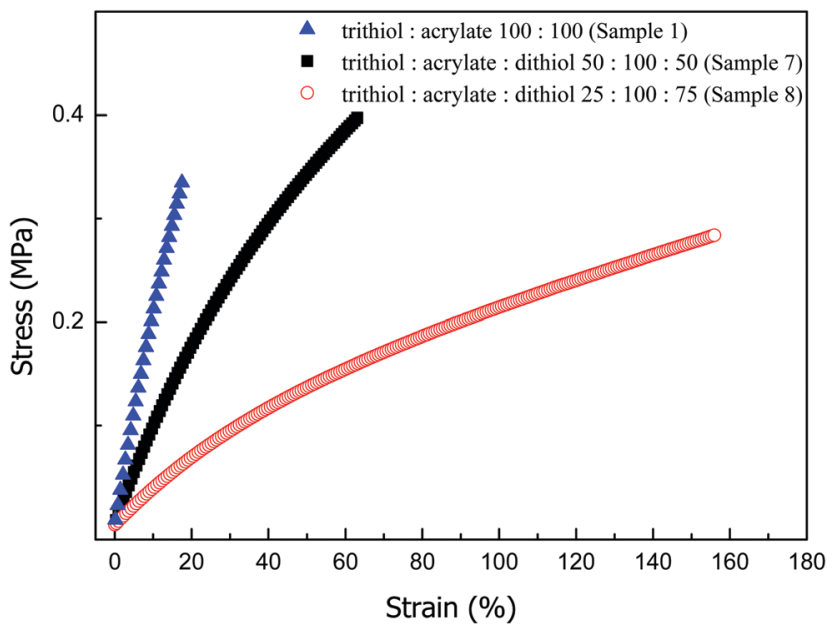

Fig. 6 Representative experimental stress-strain curves for each ionogel formed by replacing trithiol crosslinker with chain extender dithiol. Labels indicate molar percent of functional groups from trithiol, acrylate and dithiol. The detailed composition of each material can be found in Table 1.

All the ionogels resulting from this novel thiol acrylate Michael addition polymerization in ionic liquid have a high ionic conductivity (in the range of $10^{-4} \mathrm{~S} \mathrm{~cm}^{-1}$ ) even though the loading of ionic liquid is only $50 \mathrm{wt} \%$ (Table 1). This interesting polymerization reaction also offers unique processing advantages over other polymerization methods. Specifically, the thiol acrylate Michael addition polymerization is unaffected by oxygen as it proceeds via an ionic mechanism rather than radical initiated mechanism. The lack of oxygen inhibition provides a less complex procedure especially for thin polymer film preparation. These unique aspects of the thiol acrylate Michael addition reaction make it advantageous for fabricating micropatterns and micro-structures for instance for microfluidics. ${ }^{19}$ Therefore, micro-patterning of thiol acrylate based ionogel was investigated using two complementary micro-patterning techniques: soft imprinting lithography and standard photolithography.

Soft imprint lithography is a well-known, versatile technique for fabricating or replicating microstructures using moulds from elastomeric materials, most notably PDMS. ${ }^{27}$ Here, patterned PDMS was employed as the template to pattern the surface of thiol acrylate based ionogel. Ionogel solution was casted on the patterned PDMS, followed by covering with a glass plate. Next the ionogel was cured at room temperature for overnight and peeled off from the substrate, resulting in a micro-patterned ionogel. Fig. 7a and b show microscope images of the obtained patterned ionogel films (sample no. 2) that show well-defined edge profiles, demonstrating that a high fidelity was achieved in this process.

For standard photolithographic patterning, TEA was replaced with photo base generator to achieve a photopolymerizable thiol acrylate system by triggering the release of base for catalyzing the thiol acrylate Michael addition from UV irradiation. The polymer electrolyte solution (sample no. 2) was first spin coated on a gold coated silicon wafer and the sample was then irradiated through a photomask with a UV intensity of $10 \mathrm{~mW} \mathrm{~cm}^{-2}$ for 120 seconds. The development of the micro-patterns was done by rinsing the non-crosslinked materials on wafers where there was no UV illumination. Water was used as the development solvent to keep the ionic liquid inside the micro-patterns as water and the ionic liquid are immiscible. Fig. 7c and d shows the obtained micropatterns and their thickness profiles. Without any optimization, polymer patterns with a lateral structure size of $100 \mu \mathrm{m}$ and thickness of around $20 \mu \mathrm{m}$ were obtained. This clearly demonstrates the excellent micro-patternability of the polymer electrolyte solution using thiol acrylate Michael reaction.

As mentioned previously, thiol acrylate Michael addition provides a convenient way to prepare ionogels with tuneable surface properties, either a thiol rich surface or an acrylate rich surface. The density of these unreacted groups presented on the surfaces of ionogel can be controlled by the degree of off-stoichiometry and these groups are evenly distributed over the entire surface. In principle, two ionogel films with different excess groups can be covalently bonded using the same chemistry as the catalyst needed for further thiol acrylate Michael addition is still available on this truly reactive surface. ${ }^{19}$ However, two solid surfaces are difficult to conform to each other due to micro- and nano-irregularities, leading to inefficient bonding in the interface. ${ }^{28}$ In addition, bonding two different surfaces together would result in a non-uniform layered structure with two different mechanical properties. Here we present a different way to bond films with the same excess functional groups together to form uniform ionogel structures using the thiol acrylate mixture as a liquid adhesive. As a first example, a tube structure was constructed by rolling a thiol rich ionogel film together and the edges were bonded by the thiol rich electrolyte precursor mixture as the adhesive. The liquid state of the adhesive mixture in the beginning of the curing process made it easy to apply on the solid surface and bring two solid surfaces in tight contact. A strong adhesion was achieved as excess functional groups on surfaces participate in the adhesive curing process to form covalent bonds between layers through the same thiol acrylate Michael addition reaction. A uniform tube structure was therefore built as the adhesive is made from the same materials that is used to prepare the ionogel film.

The tube structure is further utilized to fabricate an ionic tubular actuator as illustrated in Fig. 8. An ionogel film from a formulation with $25 \%$ excess of thiols groups was first prepared to form the body of the actuator and to function as the ion source for ions transfer during the actuation. Conducting polymer PEDOT-PSS, which is the actuating material, was patterned as lines on both sides of the ionogel film using a PDMS mould. After the aqueous PEDOT-PSS solution had dried, the ionogel film with patterned PEDOT-PSS lines was rolled and bonded with the ionic conducting adhesive on the edges. This resulted in a compliant and flexible ionogel tube structure with a $4 \mathrm{~mm}$ small diameter. This method allowed us to fabricate two independent trilayer actuators, that are patterned both on the inside and outside of a soft tubular structure, which is otherwise extremely difficult to obtain by moulding and laser patterning. The two actuators could be individually controlled and allowed the tube to 
(a)

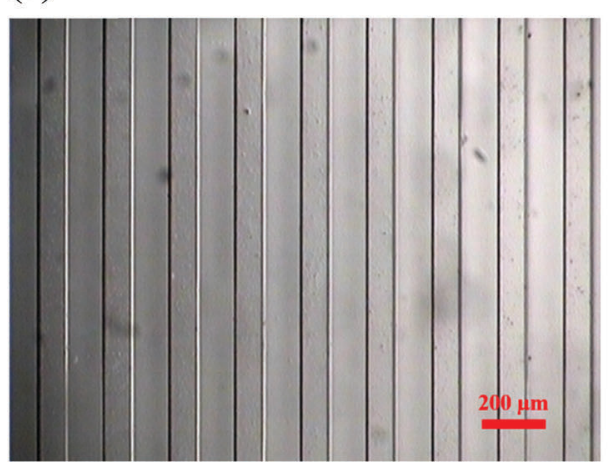

(c) (b)

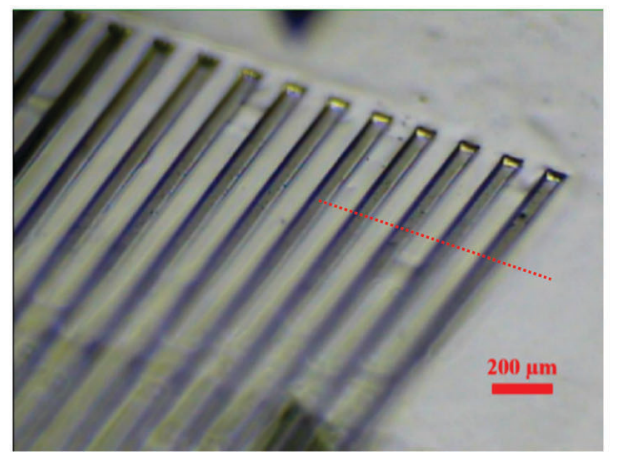

(d)
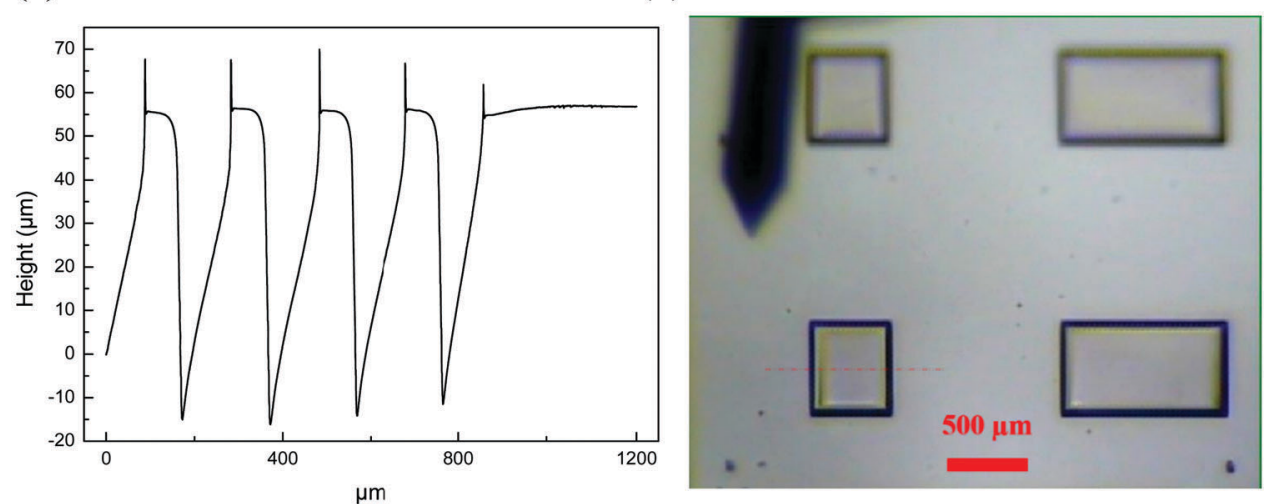

(e)

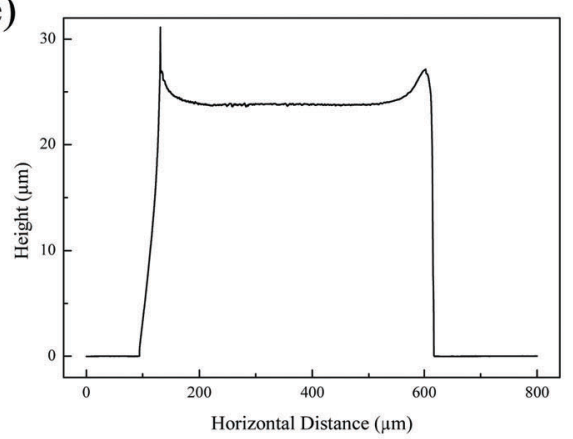

(g)

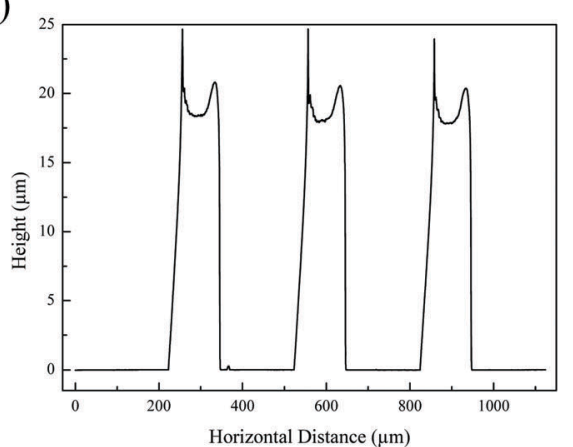

(f)

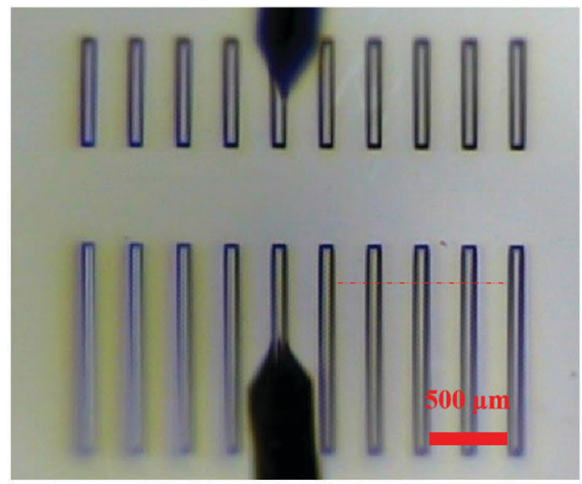

Fig. 7 Microscope images and thickness profiles for the obtained ionogel micro-patterns from soft imprinting lithography (a, top view; $b$, tilt view; $c$, thickness profile) and from standard photolithography ( $d$ and e, square patterns and thickness profile; $f$ and $g$, line patterns and thickness profile).

bend in different directions on command. Fig. 9 shows frame grabs of the motion (and the actuation video can be found in the supporting material). Such tubular structures with individually controlled actuators are interesting to use biomedical devices, especially in positioning scanners and steerable catheters and guide wires. ${ }^{29,30}$ 


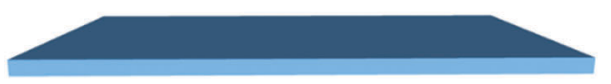

(1) Preparation of solid polymer electrolyte film with thiol rich surface

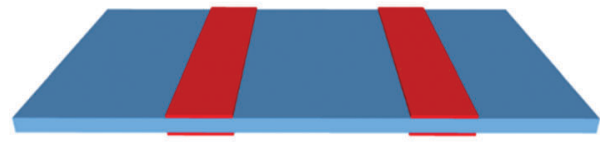

(3) Pattern the solid polymer electrolyte with PEDOT-PSS on both sides and apply electrolyte adhesive on the edges

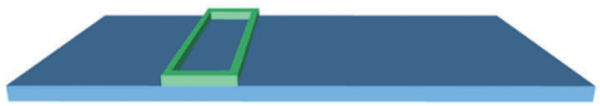

(2) Pour PEDOT-PSS solution into the PDMS mold to obtain PEDOT-PSS electrode

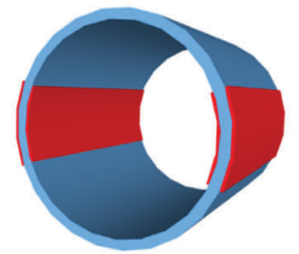

(4) Roll the film and bond the edges together into a tube to form tube actuator

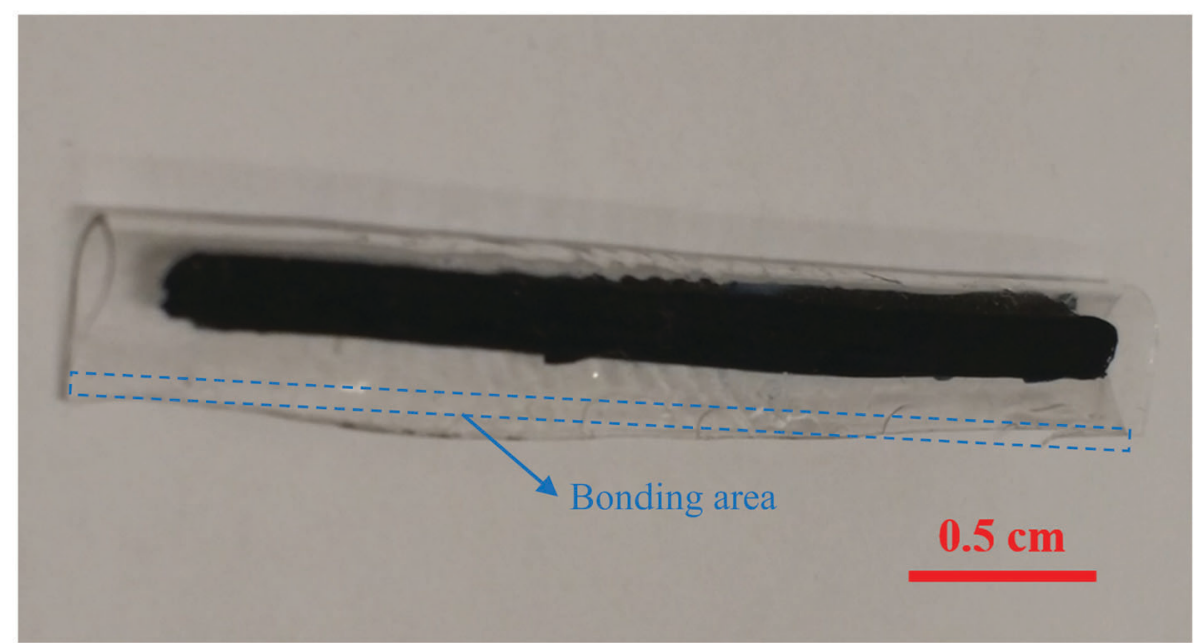

Fig. 8 (a1-a4) The schematic for procedures of tube actuator fabrication and (b) the as-prepared tube actuators.
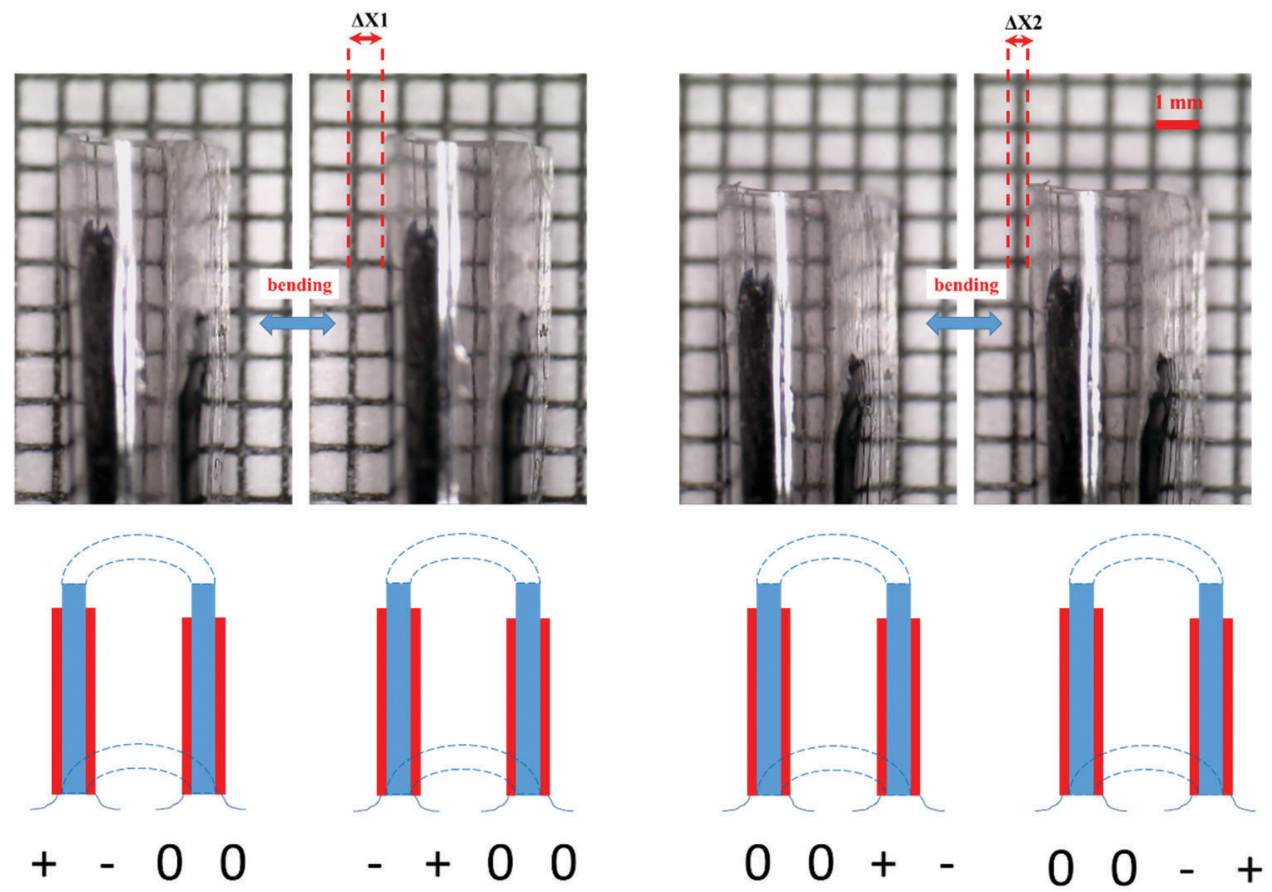

Fig. 9 Actuation movement of a tube actuator system with two independently controlled trilayer actuators, by either addressing (a) the left tri-layer actuator or (b) the right actuator. The sketches under the photos illustrate how the potential (2.0 V) was applied. 


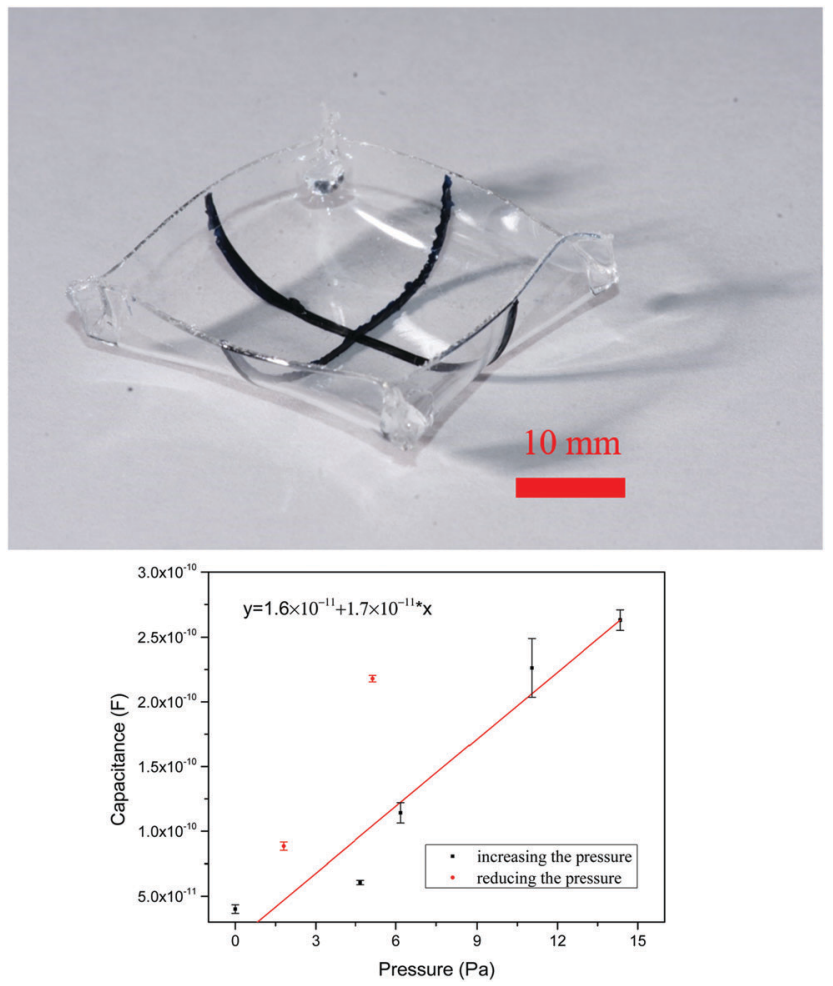

Fig. 10 (a) Photograph of ionogel with a "box" structure with 2 electrode lines forming a taxel inside the box. (b) The plot of capacitance versus the applied pressure. The line is a linear fitting of the increasing pressure points.

As a second demonstration of the versatility of this new technique, we created a 3-dimensional box with two patterned, perpendicularly aligned electrodes that form a taxel (a pressure sensor) inside the box. Like in the tubular structure, first the ionogel film was formed and the PEDOT lines were patterned using the PDMS mould, one on the outside/bottom and one on the inside/top of the ionogel. Next the box was constructed by folding the four edges and bonding them using the thiolacrylate adhesive. Fig. 10a shows the resulting structure. To illustrate an alternative use, we used this pattern as a flexible taxel, that could be formed into any 3D structure. Using a movable electrode pin an increasing force was applied and the resulting capacitance was read out (Fig. S5 and S6b, ESI $\dagger$ ). The capacitance showed a linear response (Fig. 10b and Fig. S4c, ESI $\dagger$ ) to the applied pressure. This illustrates its possibilities for flexible arrays for haptic input or flexible force/pressure sensor arrays of arbitrary shapes.

\section{Conclusion}

A new ionogel was successfully prepared using the thiol acrylate Michael addition reaction in the presence of ionic liquid. Polymerization kinetics study showed that the ionic liquid not only acts as an ion source but also a co-catalyst for thiol acrylate network formation. This leads to an extremely high functional group conversion in a very short time, which is otherwise a slow reaction in TEA catalyzed thiol-acrylate reactions. By taking advantage of this efficient stoichiometric reaction and kinetics difference, ionogels with tuneable surface and mechanical properties were achieved in three different ways: (1) off-stoichiometry, (2) methacrylate addition, and (3) dithiol addition. Off-stoichiometric formulations lead to an ionogel with remaining unreacted functional groups both in the bulk and on the surface. The Young's modulus could be varied from 2.1 MPa to 0.43 MPa by changing the acrylate and thiol ratios. By using PEGMA the Young's modulus could be altered from 2.1 MPa to 0.9 MPa due to the creation of dangling chains. By using dithiols as chain extenders, the Young's modulus was varied from 2.1 MPa to 0.3 MPa and the strain at break increased from $17 \%$ to $155 \%$. The ionogel could also be micro-patterned using both photolithography by adding a photobase generator and soft imprinting lithography.

These flexible ionogels films also exhibit remaining functionality. This was utilized to bond structures via the same polymerization technique by utilizing the electrolyte precursor mixture as ionic conducting adhesive to form complex 3-dimensional structures. Excellent bonding was achieved as the reactive groups on the surface participate in the adhesive curing process. To illustrate the potential of ionogel with reactive surfaces, two devices were created a tube actuator and box with a flexible electrode forming a taxel. The tube actuator comprised two fully independent ionic trilayer actuators that were patterned both inside and outside on the ionogel tube, which is difficult to achieve using conventional methods, and that could be individually controlled. The box consisted of patterned pressure sensor or taxel that showed a linear response to the applied force/pressure. These demonstrations illustrate that these novel, micro-patternable and tailorable ionogels can be used in a wide range of applications in soft robotics, flexible and stretchable electronics, and medical devices for instance as soft actuators, flexible haptic arrays, and steerable catheters.

\section{Materials and methods}

\section{Materials}

Trimethylolpropane tris(3-mercoptopropianate) (TT), poly(ethylene glycol)diacrylate (PEGDA, $M_{\mathrm{n}}=700$ ), poly(ethylene glycol)methacrylate (PEGMA, $M_{\mathrm{n}}=500$ ), 1-ethyl-3-methylimidazolium bis(trifluoromethylsulfonyl)imide (EMIM TFSI) and triethylamine (TEA) were purchased from Sigma-Aldrich. 2-(9-Oxoxanthen-2yl)propionic acid 1,5,7-triazabicyclo[4.4.0]dec-5-ene salt (photobase generator, PBG) and 1,4-butanediol bis(thioglycolate)(dithiol) were purchased from TCI Chemicals. PEDOT-PSS (PH1000) was purchased from Heraeus. All chemicals were used without further purification. The chemical structures of the materials are shown in Fig. 1.

\section{Preparation of ionogel films and their characterization}

The ionogel films studied in this work were prepared by mixing the thiol, acrylate monomers, TEA and ionic liquid in a vial according to the formulations shown in Table 1 . The TEA amount in all samples is $1 \mathrm{wt} \%$ relative to the weight of thiol and acrylate monomers. The weight percentage of ionic liquid is $50 \mathrm{wt} \%$ relative to the total mixture weight. The electrolyte 
precursor mixtures were then poured onto glass plates and sandwiched by a top glass plate with shims to control the thickness to $\sim 0.5 \mathrm{~mm}$. All reactions were performed at room temperature under normal atmosphere. Free-standing ionogel films were obtained after the polymer electrolyte solution achieved a complete conversion. The polymerization kinetics and functional group conversion were studied by ATR-FTIR spectroscopy using a Tensor 27 (Bruker) FT-IR instrument equipped with an ATR accessory unit. Changes in peak intensity of thiol groups at $2570 \mathrm{~cm}^{-1}$ and acrylate groups at $810 \mathrm{~cm}^{-1}$ were monitored as a function of irradiation time. ${ }^{31}$ Mechanical property measurements were evaluated on a Dynamic Mechanical Analyzer instrument (TA, Q800) in tensile mode with a frequency of $1 \mathrm{~Hz}$ and strain rate of $20 \% \mathrm{~min}^{-1}$ at room temperature. Electrochemical Impedance Spectroscopy (EIS) of ionogels was measured in the frequency range $1 \mathrm{MHz}$ to $0.01 \mathrm{~Hz}$, at 10 points per decade, with an oscillation potential of $10 \mathrm{mV}$ around $0 \mathrm{~V}$ using a VSP potentiostat (Biologic SA) at room temperature. The ionic conductivity was calculated from EIS results using the following equation

$$
\sigma=\frac{1}{Z} \frac{d}{S}
$$

where $Z$ is the real part of the complex impedance (ohms), $d$ is the thickness of the sample $(\mathrm{cm})$, and $S$ is the sample area between the two electrodes $\left(\mathrm{cm}^{2}\right){ }^{14}$

\section{Micro-patterning of ionogels by standard photolithography and soft imprinting lithography}

Patternability of ionogel was demonstrated using thiol acrylate formulation with $25 \%$ excess thiol groups. The procedures for soft imprinting lithographic patterning is shown as follows. Soft PDMS template was first prepared from SU-8 2010 patterns on a silicon wafer as described in ref. 27. The polymer electrolyte solution was drop casted on PDMS patterns and covered with a glass slide. After complete curing at room temperature, the patterned ionogel films were obtained by peeling it off from the patterned PDMS. For standard photolithographic patterning, TEA was replaced with photobase generator (PBG) to achieve a photopolymerizable thiol acrylate system. The amount of PBG is $1 \mathrm{wt} \%$ and $50 \mu \mathrm{L}$ of ethanol is added to facilitate the dissolution of PBG in the mixture. The resulting electrolyte precursor mixture was spun onto a gold coated silicon wafer at $800 \mathrm{rpm}$ with an acceleration of $300 \mathrm{rpm} \mathrm{s}^{-1}$ for 30 seconds. Patterned UV illumination was performed by using a mask aligner (SUSS MicroTec) with UV intensity of $10 \mathrm{~mW} \mathrm{~cm}^{-2}$ for 120 seconds. The uncured materials in the unexposed areas were washed off with deionized water. The thickness profile for these micro-patterns was measured with a Veeco Dektak 6M surface profilometer.

\section{Tube actuator fabrication and characterization}

Conducting polymer PEDOT-PSS was used as the actuating material in ionic tube actuator fabrication. In order to increase the electrical properties of PEDOT-PSS, DMSO (Sigma-Aldrich) solvent ( $5 \%$ by volume) was added to aqueous PEDOT-PSS (Heraeus PH1000) solution. ${ }^{32}$ A PDMS mould with line patterns (around $3.5 \mathrm{~cm} \times 0.2 \mathrm{~cm}$ ) to define the size of the PEDOT-PSS electrodes was pressed on the ionogel film prepared from formulation with $25 \%$ excess thiol groups. The PEDOT-PSS solution was then dropped into the channels formed by the PDMS mould on top of the ionogel films. Patterned PEDOT-PSS electrodes were then obtained after PEDOT-PSS had dried in the room temperature for overnight and the PDMS moulds was removed. The same procedure was performed on the other side of ionogel film, with the PDMS mould aligned to the PEDOT-PSS electrode pattern, to achieve a trilayer actuator structure. ${ }^{33,34}$ Tube actuators were finally constructed by rolling the patterned ionogels and bonding the sides together using electrolyte precursor mixture with the same formation of ionogel film as adhesive. The actuators were subjected to a step potential of $2 \mathrm{~V}$ for actuation characterization. The procedures of fabrication ionic tube actuator are summarized in Fig. 7.

\section{Box fabrication}

The box was fabricated similarly to the tube. However, the PEDOT electrodes were perpendicularly aligned and instead of rolling the film, the four sides were folded together and bonded at the edges.

\section{Capacitance measurements}

The capacitance of the pressure sensor was measured using a precision LCR (inductance, capacitance, resistance) meter (Agilent 4284A) under ambient conditions. The frequency was fixed at 1.0 MHz with a bias voltage of $0 \mathrm{~V}$. A manual controlled manipulator was set up with the LCR meter to measure the capacitance dependence with force. The force applied on the sample was determined by using a balance (PCB1000-2, KERN) which was placed under the bottom electrode and the force was calculated $(F=m \times g)$. The applied pressure was calculated from the cross-sectional area of the electrode pin $\left(3.3 \mathrm{~mm}^{2}\right)$. A photograph and schematics of the capacitance force measurement setup can be found in Fig. S4 in the ESI. $\dagger$

\section{Conflicts of interest}

The authors declare that they have no competing interests.

\section{Acknowledgements}

The research was supported by European Union's Horizon 2020 research and innovation program under the Marie Sklodowska Curie grant agreement no. 641822, the Swedish Research Council (VR 2014-3079). In addition, the authors would like to thank Dr Mike Andersson for the help in the measurement of capacitance and Prof Marc in het Panhuis, University of Wollongong, for scientific discussions.

\section{References}

1 B. Chen, J. J. Lu, C. H. Yang, J. H. Yang, J. Zhou, Y. M. Chen and Z. Suo, ACS Appl. Mater. Interfaces, 2014, 6, 7840-7845.

2 M. A. B. H. Susan, T. Kaneko, A. Noda and M. Watanabe, J. Am. Chem. Soc., 2005, 127, 4976-4983. 
3 S. H. Kim, K. Hong, W. Xie, K. H. Lee, S. Zhang, T. P. Lodge and C. D. Frisbie, Adv. Mater., 2013, 25, 1822-1846.

4 W. Zuo, R. Li, C. Zhou, Y. Li, J. Xia and J. Liu, Adv. Sci., 2017, 4, 1600539.

5 S. Ahmad and M. Deepa, Electrochem. Commun., 2007, 9, 1635-1638.

6 A. Maziz, C. Plesse, C. Soyer, C. Chevrot, D. Teyssié, E. Cattan and F. Vidal, Adv. Funct. Mater., 2014, 24, 4851-4859.

7 Rahul, B. Bhattacharya, P. K. Singh, R. Singh and Z. H. Khan, Int. J. Hydrogen Energy, 2016, 41, 2847-2852.

8 F. Opekar and K. Štulík, Anal. Chim. Acta, 1999, 385, 151-162.

9 W. Liu, M.-S. Song, B. Kong and Y. Cui, Adv. Mater., 2017, 29, 1603436.

10 M. Armand, F. Endres, D. R. MacFarlane, H. Ohno and B. Scrosati, Nat. Mater., 2009, 8, 621-629.

11 J.-H. Choi, Y. Gu, K. Hong, W. Xie, C. D. Frisbie and T. P. Lodge, ACS Appl. Mater. Interfaces, 2014, 6, 19275-19281.

12 S. K. Lee, S. M. Kabir, B. K. Sharma, B. J. Kim, J. H. Cho and J. H. Ahn, Nanotechnology, 2014, 25, 014002.

13 K. Fujii, H. Asai, T. Ueki, T. Sakai, S. Imaizumi, U.-i. Chung, M. Watanabe and M. Shibayama, Soft Matter, 2012, 8, 1756-1759.

14 L. J. Goujon, A. Khaldi, A. Maziz, C. Plesse, G. T. M. Nguyen, P.-H. Aubert, F. Vidal, C. Chevrot and D. Teyssié, Macromolecules, 2011, 44, 9683-9691.

15 A. B. Lowe, Polym. Chem., 2014, 5, 4820-4870.

16 D. P. Nair, M. Podgórski, S. Chatani, T. Gong, W. Xi, C. R. Fenoli and C. N. Bowman, Chem. Mater., 2014, 26, 724-744.

17 N. G. Moon, R. J. Mondschein and T. E. Long, Polym. Chem., 2017, 8, 2598-2608.

18 B. D. Mather, K. Viswanathan, K. M. Miller and T. E. Long, Prog. Polym. Sci., 2006, 31, 487-531.
19 C. O. Bounds, J. Upadhyay, N. Totaro, S. Thakuri, L. Garber, M. Vincent, Z. Huang, M. Hupert and J. A. Pojman, ACS Appl. Mater. Interfaces, 2013, 5, 1643-1655.

20 W. Xi, H. Peng, A. Aguirre-Soto, C. J. Kloxin, J. W. Stansbury and C. N. Bowman, Macromolecules, 2014, 47, 6159-6165.

21 W. Xi, C. Wang, C. J. Kloxin and C. N. Bowman, ACS Macro Lett., 2012, 1, 811-814.

22 A. Sarkar, S. R. Roy and A. K. Chakraborti, Chem. Commun., 2011, 47, 4538-4540.

23 B. C. Ranu, S. Banerjee and R. Jana, Tetrahedron, 2007, 63, 776-782.

24 B. C. Ranu and S. S. Dey, Tetrahedron, 2004, 60, 4183-4188.

25 M. Meciarova, S. Toma and P. Kotrusz, Org. Biomol. Chem., 2006, 4, 1420-1424.

26 S. Chatani, D. P. Nair and C. N. Bowman, Polym. Chem., 2013, 4, 1048-1055.

27 Y. Xia and G. M. Whitesides, Annu. Rev. Mater. Sci., 1998, 28, 153-184.

28 C. F. Carlborg, T. Haraldsson, K. Oberg, M. Malkoch and W. van der Wijngaart, Lab Chip, 2011, 11, 3136-3147.

29 M. Farajollahi, V. Woehling, C. Plesse, G. T. M. Nguyen, F. Vidal, F. Sassani, V. X. D. Yang and J. D. W. Madden, Sens. Actuators, A, 2016, 249, 45-56.

$30 \mathrm{~J}$. Leger, M. Berggren and S. Carter, IONTRONICS: ionic carriers in organic electronic materials and devices, CRC Press, 2017.

31 E. R. Beckel, J. Nie, J. W. Stansbury and C. N. Bowman, Macromolecules, 2004, 37, 4062-4069.

32 H. Shi, C. Liu, Q. Jiang and J. Xu, Adv. Electron. Mater., 2015, 1, 1500017.

33 H. Okuzaki, S. Takagi, F. Hishiki and R. Tanigawa, Sens. Actuators, B, 2014, 194, 59-63.

34 N. Terasawa and K. Asaka, Langmuir, 2016, 32, 7210-7218. 